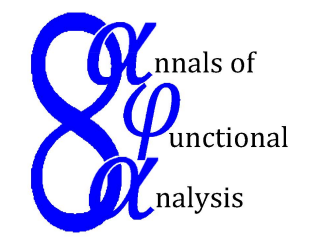

Ann. Funct. Anal. 6 (2015), no. 4, 179-190

http://doi.org/10.15352/afa/06-4-179

ISSN: 2008-8752 (electronic)

http://projecteuclid.org/afa

\title{
INVARIANT SUBSPACES OF COMPOSITION OPERATORS ON A HILBERT SPACE OF DIRICHLET SERIES
}

\author{
MAOFA WANG AND XINGXING YAO* \\ Communicated by M. Ptak
}

\begin{abstract}
In this paper, we study invariant subspaces of composition operators on the Hilbert space of Dirichlet series with square summable coefficients. The structure of invariant subspaces of a composition operator is characterized, and the strongly closed algebras generated by some composition operators with irrational symbols are shown to be reflexive. As an application, we provide a criterion for composition operators with certain symbols not to be algebraic.
\end{abstract}

\section{INTRODUCTION}

For $H$ a space of analytic functions on a region $G \subseteq \mathbb{C}$ and $\varphi$ an analytic map of $G$ into itself, the composition operator $C_{\varphi}$ is a linear operator defined by

$$
C_{\varphi} f=f \circ \varphi, f \in H .
$$

Broadly, one is interested in extracting properties of $C_{\varphi}$ acting on $H$ (boundedness, compactness, spectral properties, etc.) from function theoretic properties of $\varphi$ and vice versa. We refer to monographs by Cowen-MacCluer [4], Shapiro [15] and Zhu [18] for general information of composition operators on the open unit disc $\mathbb{D}$ of the complex plane $\mathbb{C}$. Recently, an extensive study of composition operators was carried out on various spaces of Dirichlet series (see $[1,2,3,6,12,16]$ ).

Let $\mathcal{H}$ be the Hilbert space of Dirichlet series with square summable coefficients:

$$
\mathcal{H}=\left\{f(s)=\sum_{n=1}^{\infty} a_{n} n^{-s}:\|f\|=\left(\sum_{n=1}^{\infty}\left|a_{n}\right|^{2}\right)^{1 / 2}<\infty\right\} .
$$

Date: Received: Feb. 9, 2015; Accepted: Apr. 29, 2015.

* Corresponding author.

2010 Mathematics Subject Classification. Primary 47B33; Secondary 30D55, 46E15.

Key words and phrases. Dirichlet series, composition operator, invariant subspace, algebraic operator. 
By the Cauchy-Schwarz inequality, the functions in $\mathcal{H}$ are all analytic on the half-plane $\mathbb{C}_{1 / 2}$ (where, for $\theta \in \mathbb{R}, \mathbb{C}_{\theta}=\{s \in \mathbb{C}: \operatorname{Re} s>\theta\}$ and $\mathbb{C}_{+}=\mathbb{C}_{0}$ ). In the process, we also encounter the space $\mathcal{D}$ of functions $f$, which in some (possibly remote) half-plane admit representation by a convergent Dirichlet series $f(s)=\sum_{n=1}^{\infty} a_{n} n^{-s}$. We also recall that a series in $\mathcal{D}$ actually converges absolutely in the half-plane one unit to the right of the half-plane of convergence.

The following comes from $[6,12]$, which characterizes the boundedness of composition operators on $\mathcal{H}$.

Theorem A. An analytic function $\Phi: \mathbb{C}_{1 / 2} \rightarrow \mathbb{C}_{1 / 2}$ determines a bounded composition operator $C_{\Phi}: \mathcal{H} \rightarrow \mathcal{H}$ if and only if $\Phi(s)=c_{0} s+\varphi(s)$, where $c_{0}$ is a non-negative integer, and $\varphi(s)=\sum_{n=1}^{\infty} c_{n} n^{-s} \in \mathcal{D}$ converges uniformly in $\mathbb{C}_{\epsilon}$ for every $\epsilon>0$ with the following properties:

(a) If $c_{0}=0$, then $\varphi\left(\mathbb{C}_{+}\right) \subseteq \mathbb{C}_{1 / 2}$.

(b) If $c_{0} \geq 1$, then either $\varphi \equiv 0$ or $\varphi\left(\mathbb{C}_{+}\right) \subseteq \mathbb{C}_{+}$.

It is convenient to call $\Phi$ a $c_{0}$-symbol if $\Phi: \mathbb{C}_{1 / 2} \rightarrow \mathbb{C}_{1 / 2}$ is analytic and satisfies the above conditions such that $C_{\Phi}$ is bounded on $\mathcal{H}$.

We know one of the most famous unsolved problems in analysis is the invariant subspace problem: does every bounded linear operator on an infinite dimensional Hilbert space have a non-trivial invariant subspace? In recent decades, the invariant subspaces of composition operators acting on the Hardy-Hilbert space $H^{2}(\mathbb{D})$ have been extensively studied. Particularly, the authors [10] showed that every hyperbolic composition operator on $H^{2}(\mathbb{D})$ is cosubnormal and has universal translates. This allows us to formulate the general invariant subspace problem in terms of invariant subspaces of certain composition operators. Because of this close relationship, the invariant subspaces of invertible composition operators have been studied in [9], and Mahvidi studied the structure of invariant subspaces of a general composition operator in [8].

The purpose of this paper is to generalize these results mentioned above from the classical Hardy-Hilbert space $H^{2}(\mathbb{D})$ to the space $\mathcal{H}$ of Dirichlet series. Section 2 includes some background materials needed in the sequel and some basic facts on the dynamical system of $c_{0}$-symbols, which are very different from the classical case. In Section 3, we consider the structure of invariant subspaces of composition operators. The reflexivity of algebras generated by composition operators and the non-existence of non-trivial algebraic composition operators are discussed in Section 4.

\section{Preliminaries}

Let $H$ be a Hilbert space and $B(H)$ the algebra of all bounded linear operators on $H$. Recall that a closed subspace $M$ of $H$ is called an invariant subspace of $A \in B(H)$, if $A x \in M$ for every $x \in M$ (i.e., $A M \subseteq M)$. For $A \in B(H)$, we denote the lattice of all invariant subspaces of $A$ by Lat $A$. For $\mathcal{U} \subseteq B(H)$, we set

$$
\operatorname{Lat} \mathcal{U}:=\bigcap_{A \in \mathcal{U}} \operatorname{Lat} A
$$


An algebra $\mathcal{U} \subseteq B(H)$ is said to be reflexive, if for any $B \in B(H)$ the inclusion Lat $\mathcal{U} \subseteq$ Lat $B$ implies $B \in \mathcal{U}$. Further, if $\mathcal{S}$ is any collection of subspaces of $H$, we define $\operatorname{Alg} \mathcal{S}:=\{B \in B(H): \mathcal{S} \subseteq$ Lat $B\}$. Then the algebra $\mathcal{U}$ is reflexive if and only if $\mathcal{U}=\operatorname{Alg} \operatorname{Lat} \mathcal{U}$. Reflexive algebras are associated extensively to the transitive and reductive algebra problems. For more details, see [13].

We know that $\mathcal{H}$ is a reproducing kernel Hilbert space and the reproducing kernel at $w$ in $\mathbb{C}_{1 / 2}$ is given by $K_{w}(s)=\sum_{n=1}^{\infty} n^{-\bar{w}} n^{-s}$ for $\operatorname{Re} s>1 / 2$.

If we denote by $C_{\Phi}^{*}$ the adjoint of $C_{\Phi}$ whenever it is bounded, then for $w \in \mathbb{C}_{1 / 2}$ we have

$$
C_{\Phi}^{*}\left(K_{w}\right)=K_{\Phi(w)}
$$

Let $\Phi$ be an analytic self-map of $\mathbb{C}_{1 / 2}$. For all $n \in \mathbb{N}$ we denote the $n$-th iterate of $\Phi$ by $\Phi_{n}$, that is $\Phi_{n}:=\Phi \circ \cdots \circ \Phi$. For $n=0$ we set $\Phi_{0}:=i d$, the identity function of $\mathbb{C}_{1 / 2}$.

The followings are from [2] and [6] respectively.

Lemma 2.1. Let $m$ be a positive integer, and $f(s)=\sum_{n=m}^{\infty} a_{n} n^{-s} \in \mathcal{D}$. Then $m^{s} f(s) \rightarrow a_{m}$ uniformly as $\operatorname{Re} s \rightarrow+\infty$.

Lemma 2.2. Let $\Phi(s)=c_{0} s+\varphi(s)$ be an analytic self-map of $\mathbb{C}_{+}$. If $\Phi(s) \neq s+i \tau$, $\tau \in \mathbb{R}$, then there exist $\eta>0$ and $\epsilon>0$ such that $\Phi\left(\mathbb{C}_{1 / 2-\epsilon}\right) \subseteq \mathbb{C}_{1 / 2+\eta}$.

We next consider the iterates of $c_{0}$-symbols, which are very different from the classical case (see [4]). Therefore, we have to use essentially different methods to study invariant subspaces of composition operators acting on $\mathcal{H}$.

The following corollary shows that for any $\lambda \in \mathbb{C}_{1 / 2}$, the sequence $\left\{\Phi_{n}(\lambda)\right\}$ never satisfies the "Blaschke" condition.

Corollary 2.3. Let $\Phi(s)=c_{0} s+\varphi(s)$ be an analytic self-map of $\mathbb{C}_{+}$. Then there does not exist $\lambda \in \mathbb{C}_{1 / 2}$ such that $\operatorname{Re} \Phi_{n}(\lambda) \rightarrow \frac{1}{2}$ as $n \rightarrow \infty$.

The next concerns the dynamical system of $c_{0}$-symbols in $\mathbb{C}_{1 / 2}$.

Theorem 2.4. If a $c_{0}$-symbol $\Phi$ is neither a constant nor the identity, then the set $\left\{\Phi_{n}(w)\right\}_{n=0}^{\infty}$ has no limit points other than the infinity point in $\mathbb{C}_{1 / 2}$ for each $w \in \mathbb{C}_{1 / 2}$.

Proof. Since it is trivial for the case where $\Phi(s)=s+i \tau$ with $\tau \in \mathbb{R} \backslash\{0\}$, then we assume that $\Phi(s) \neq s+i \tau(\tau \in \mathbb{R})$ is a $c_{0}$-symbol. Here we argue by contradiction. To this end, we assume that the set $\left\{\Phi_{n}\left(w_{0}\right)\right\}_{n=0}^{\infty}$ has a finite limit point in $\mathbb{C}_{1 / 2}$ for some point $w_{0} \in \mathbb{C}_{1 / 2}$. Let $g \in \mathcal{H}$ be such that $g \perp \overline{\operatorname{span}}\left\{\left(C_{\Phi}^{*}\right)^{n} K_{w_{0}}: n \geq 0\right\}$. Then it is clear that

$$
0=\left\langle g,\left(C_{\Phi}^{*}\right)^{n} K_{w_{0}}\right\rangle=\left\langle g, K_{\Phi_{n}\left(w_{0}\right)}\right\rangle=g\left(\Phi_{n}\left(w_{0}\right)\right),
$$

that is, the analytic function $g$ has zeros $\Phi_{n}\left(w_{0}\right)(n \geq 0)$. Since $\Phi$ is a nonconstant analytic self-map of $\mathbb{C}_{1 / 2}$, then $g \equiv 0$ by the identity theorem. So we have $\overline{\operatorname{span}}\left\{\left(C_{\Phi}^{*}\right)^{n} K_{w_{0}}: n \geq 0\right\}=\mathcal{H}$.

It follows from Lemma 2.2 that there exists $\eta>0$ such that $\Phi\left(\mathbb{C}_{1 / 2}\right) \subseteq \mathbb{C}_{1 / 2+\eta}$. Then $\Phi_{n}\left(w_{0}\right) \in \mathbb{C}_{1 / 2+\eta}$ for all $n \geq 1$. Thus we can choose some $w_{1} \in \mathbb{C}_{1 / 2}$ such that $1 / 2<\operatorname{Re} w_{1}<1 / 2+\epsilon$ for some positive number $\epsilon<\eta$ and $\operatorname{Re} w_{1} \neq \operatorname{Re} w_{0}$. 
Since $K_{w_{1}} \in \mathcal{H}=\overline{\operatorname{span}}\left\{\left(C_{\Phi}^{*}\right)^{n} K_{w_{0}}: n \geq 0\right\}=\overline{\operatorname{span}}\left\{K_{\Phi_{n}\left(w_{0}\right)}: n \geq 0\right\}$, then there must exist a sequence $\left\{g_{N}\right\}_{N \geq 0}$ with $g_{N}(s)=\sum_{\ell=0}^{m(N)} a_{\ell}^{(N)} K_{\Phi_{\ell}\left(w_{0}\right)}(s)$, such that $g_{N} \rightarrow K_{w_{1}}$ in $\mathcal{H}$ as $N \rightarrow \infty$. Note that

$$
g_{N}(s)=\sum_{\ell=0}^{m(N)} a_{\ell}^{(N)} \sum_{n=1}^{\infty} n^{-\overline{\Phi_{\ell}\left(w_{0}\right)}} n^{-s}=\sum_{n=1}^{\infty}\left(\sum_{\ell=0}^{m(N)} a_{\ell}^{(N)} n^{-\overline{\Phi_{\ell}\left(w_{0}\right)}}\right) n^{-s}
$$

for each $N \geq 0$. Thus we get that $\sum_{\ell=0}^{m(N)} a_{\ell}^{(N)} n^{-\overline{\Phi_{\ell}\left(w_{0}\right)}} \rightarrow n^{-\overline{w_{1}}}$, that is,

$$
\sum_{\ell=0}^{m(N)} \overline{a_{\ell}^{(N)}} n^{w_{1}-\Phi_{\ell}\left(w_{0}\right)} \rightarrow 1
$$

for all $n \geq 1$, as $N \rightarrow \infty$. Note that $\operatorname{Re} w_{1} \neq \operatorname{Re} w_{0}$ and $\operatorname{Re} w_{1}-\operatorname{Re} \Phi_{\ell}\left(w_{0}\right)<$ $-(\eta-\epsilon)$ for all $\ell \geq 1$. Thus (2.1) is impossible. This contradiction completes the proof.

\section{INVARIANT SUBSPACES}

We now consider the structure of invariant subspaces of composition operators. First, we notice the following simple observation concerning the images of 1 of the adjoints of composition operators on $\mathcal{H}$.

Lemma 3.1. Let $\Phi(s)=c_{0} s+\sum_{k=1}^{\infty} c_{k} k^{-s}$ be a $c_{0}$-symbol. Then the following statements hold:

(1) If $c_{0}=0$, then $C_{\Phi}^{*} 1=\sum_{n=1}^{\infty} n^{-\overline{c_{1}}} n^{-s}$.

(2) If $c_{0} \geq 1$, then $C_{\Phi}^{*} 1=1$.

Proof. (1) If $c_{0}=0$, then $\operatorname{Re} c_{1}>\frac{1}{2}$ by [6, P.319] and for each $f(s)=\sum_{n=1}^{\infty} a_{n} n^{-s} \in$ $\mathcal{H}$, we have

$$
C_{\Phi} f(s)=\sum_{n=1}^{\infty} a_{n} n^{-c_{1}} n^{-\sum_{k=2}^{\infty} c_{k} k^{-s}}=\sum_{n=1}^{\infty} a_{n} n^{-c_{1}} \prod_{k=2}^{\infty}\left(1+\sum_{j=1}^{\infty} \frac{\left(-c_{k} \log n\right)^{j}}{j !} k^{-j s}\right),
$$

which holds in the half-plane of absolute convergence of the series $\sum_{k=1}^{\infty} c_{k} k^{-s}$. Thus

$$
\left\langle C_{\Phi}^{*} 1, f\right\rangle=\left\langle 1, C_{\Phi} f\right\rangle=\overline{C_{\Phi} f(+\infty)}=\sum_{n=1}^{\infty} \overline{a_{n}} n^{-\overline{c_{1}}}=\left\langle\sum_{n=1}^{\infty} n^{-\overline{c_{1}}} n^{-s}, f\right\rangle .
$$

By the arbitrariness of $f \in \mathcal{H}$, we get that $C_{\Phi}^{*} 1=\sum_{n=1}^{\infty} n^{-\overline{c_{1}}} n^{-s}$.

(2) If $c_{0} \geq 1$, then

$$
\left\langle C_{\Phi}^{*} 1, n^{-s}\right\rangle=\left\langle 1, n^{-\Phi(s)}\right\rangle=0
$$

for all $n \geq 2$. Thus (2) is immediately obtained.

The following concerns the lattices of two composition operators.

Theorem 3.2. Let $\Phi(s)=c_{0}^{(1)} s+\sum_{k=1}^{\infty} c_{k}^{(1)} k^{-s}$ and $\Psi(s)=c_{0}^{(2)} s+\sum_{k=1}^{\infty} c_{k}^{(2)} k^{-s}$ be $c_{0}$-symbols. If Lat $C_{\Phi} \subseteq$ Lat $C_{\Psi}$, then the following statements hold:

(1) If $c_{0}^{(1)}=0$, then $\Psi(\alpha)=\alpha$, where $\alpha$ is the fixed point of $\Phi$.

(2) If $c_{0}^{(1)} \geq 1$, then $c_{0}^{(2)} \geq 1$. 
Proof. (2) follows easily from Lemma 3.1, we only need to prove (1). Suppose that $c_{0}^{(1)}=0$ and $\alpha$ is the fixed point of $\Phi$ in $\mathbb{C}_{1 / 2}$. If Lat $C_{\Phi} \subseteq$ Lat $C_{\Psi}$, then Lat $C_{\Phi}^{*} \subseteq$ Lat $C_{\Psi}^{*}$. Note that $\operatorname{span}\left\{K_{\alpha}\right\} \in$ Lat $C_{\Phi}^{*}$, then

$$
K_{\Psi(\alpha)}=C_{\Psi}^{*} K_{\alpha} \in \operatorname{span}\left\{K_{\alpha}\right\} .
$$

If $\Psi(\alpha) \neq \alpha$, then $K_{\alpha}$ and $K_{\Psi(\alpha)}$ are linearly independent. So $\Psi(\alpha)=\alpha$.

We also consider the images of two composition operators.

Theorem 3.3. Let $\Phi(s)=c_{0}^{(1)} s+\sum_{k=1}^{\infty} c_{k}^{(1)} k^{-s}$ and $\Psi(s)=c_{0}^{(2)} s+\sum_{k=1}^{\infty} c_{k}^{(2)} k^{-s}$ be $c_{0}$-symbols such that $C_{\Phi} \mathcal{H} \subseteq C_{\Psi} \mathcal{H}$. Then the following statements hold:

(1) If $c_{0}^{(1)}=0$ and either $c_{2}^{(1)}$ or $c_{3}^{(1)}$ is not zero, then $c_{0}^{(2)} \leq 1$.

(2) If $c_{0}^{(1)} \geq 1$, then $c_{0}^{(2)} \leq c_{0}^{(1)}$.

Proof. (1) Let $c_{0}^{(1)}=0$ and assume that either $c_{2}^{(1)}$ or $c_{3}^{(1)}$ is not zero. By way of contradiction, we assume that $c_{0}^{(2)}>1$, then $n^{c_{0}^{(2)}} \geq n^{2} \geq 2^{2}$ for all $n \geq 2$. Thus

$$
\left\langle\ell^{-s}, C_{\Psi}\left(n^{-s}\right)\right\rangle=\left\langle\ell^{-s}, n^{-c_{0}^{(2)} s} n^{-c_{1}^{(2)}} n^{-\sum_{k=2}^{\infty} c_{k}^{(2)} k^{-s}}\right\rangle=0
$$

for all $n \geq 1$ and $\ell=2$ or 3 . That is $\ell^{-s} \in\left(C_{\Psi} \mathcal{H}\right)^{\perp}$, then $\ell^{-s} \in\left(C_{\Phi} \mathcal{H}\right)^{\perp}$, for $\ell=2$ or 3 . However,

$$
\left\langle\ell^{-s}, C_{\Phi}\left(n^{-s}\right)\right\rangle=\left\langle\ell^{-s}, n^{-c_{1}^{(1)}} n^{-\sum_{k=2}^{\infty} c_{k}^{(1)} k^{-s}}\right\rangle=-\overline{n^{-c_{1}^{(1)}} c_{\ell}^{(1)} \log n} \neq 0
$$

for all $n>1$ and some $\ell \in\{2,3\}$, which implies that $\ell^{-s} \notin\left(C_{\Phi} \mathcal{H}\right)^{\perp}$ for $\ell=2$ or 3 . This contradiction implies that $c_{0}^{(2)} \leq 1$.

(2) If $c_{0}^{(1)} \geq 1$ and $c_{0}^{(2)}>c_{0}^{(1)}$, then $n^{c_{0}^{(2)}}>n^{c_{0}^{(1)}} \geq 2^{c_{0}^{(1)}}$ for all $n \geq 2$. Note that

$$
\left\langle 2^{-c_{0}^{(1)} s}, C_{\Psi}\left(n^{-s}\right)\right\rangle=\left\langle 2^{-c_{0}^{(1)} s}, n^{-c_{0}^{(2)} s} n^{-c_{1}^{(2)}} n^{-\sum_{k=2}^{\infty} c_{k}^{(2)} k^{-s}}\right\rangle=0
$$

for all $n \geq 1$, and

$$
\left\langle 2^{-c_{0}^{(1)} s}, C_{\Phi}\left(2^{-s}\right)\right\rangle=\left\langle 2^{-c_{0}^{(1)} s}, 2^{-c_{0}^{(1)}} s 2^{-c_{1}^{(1)}} 2^{-\sum_{k=2}^{\infty} c_{k}^{(1)} k^{-s}}\right\rangle=\overline{2^{-c_{1}^{(1)}}} \neq 0 .
$$

Since the inclusion $C_{\Phi} \mathcal{H} \subseteq C_{\Psi} \mathcal{H}$ means that $\left(C_{\Psi} \mathcal{H}\right)^{\perp} \subseteq\left(C_{\Phi} \mathcal{H}\right)^{\perp}$, then the previous two equations leads to a contradiction. So $c_{0}^{(2)} \leq c_{0}^{(1)}$, which completes the proof.

Recall that an operator is reductive if every invariant subspace for the operator is reducing. The following characterizes the reductive composition operators on $\mathcal{H}$.

Theorem 3.4. Let $\Phi(s)=c_{0} s+\sum_{k=1}^{\infty} c_{k} k^{-s}$ be a $c_{0}$-symbol. Then $C_{\Phi}: \mathcal{H} \rightarrow \mathcal{H}$ is reductive if and only if $\Phi(s)=s+c_{1}$ with $\operatorname{Re} c_{1} \geq 0$.

Proof. We first claim that $c_{0} \neq 0$. To this end, by way of contradiction, we assume that $c_{0}=0$. It is clear that $\mathbb{C} \in$ Lat $C_{\Phi}$, which implies that $C_{\Phi}^{*} 1 \in \mathbb{C}$ by the reduction of $C_{\Phi}$. Hence $\left\langle C_{\Phi}^{*} 1, n^{-s}\right\rangle=0$ for all $n \geq 2$. But $\left\langle C_{\Phi}^{*} 1, n^{-s}\right\rangle=n^{-\overline{c_{1}}} \neq 0$ for each $n \geq 2$ by Lemma 3.1. This contradiction gives the claim. 
Next we claim that $c_{0}=1$. If this is not the case, then $c_{0}>1$. Then $2^{c_{0}}>2$. It is clear that $\mathcal{L}_{n}:=\overline{\operatorname{span}}\left\{k^{-s}: k \geq n\right\} \in$ Lat $C_{\Phi}$, and then $\mathcal{L}_{n} \in$ Lat $C_{\Phi}^{*}$ for all $n \geq 1$ by the reduction of $C_{\Phi}$. But

$$
\left\langle C_{\Phi}^{*} 2^{-c_{0} s}, 2^{-s}\right\rangle=\left\langle 2^{-c_{0} s}, 2^{-\Phi(s)}\right\rangle=2^{-\overline{c_{1}}} \neq 0,
$$

which implies that $C_{\Phi}^{*} 2^{-c_{0} s} \notin \mathcal{L}_{2^{c_{0}}}$. This contradicts to $\mathcal{L}_{2^{c_{0}}} \in \operatorname{Lat} C_{\Phi}^{*}$, then $c_{0}=1$.

So we can suppose that $\Phi(s)=s+c_{1}+\sum_{k=2}^{\infty} c_{k} k^{-s}$. Since $\mathcal{L}_{n} \in$ Lat $C_{\Phi}$, and then $\mathcal{L}_{n} \in$ Lat $C_{\Phi}^{*}$ for all $n \geq 1$. Note that $\mathcal{L}_{n}^{\perp}=\operatorname{span}\left\{1,2^{-s}, \cdots,(n-1)^{-s}\right\}$ for $n \geq 2$, and $\mathcal{H}^{\perp}=\{0\}$. So $C_{\Phi}\left(m^{-s}\right) \in \operatorname{span}\left\{1,2^{-s}, \cdots,(n-1)^{-s}\right\}$ for each $n \geq 2$ and $m \in\{1,2, \cdots, n-1\}$. Note that

$$
\begin{aligned}
C_{\Phi}\left(m^{-s}\right) & =m^{-\Phi(s)}=m^{-c_{1}} m^{-s} m^{-\sum_{k=2}^{\infty} c_{k} k^{-s}} \\
& =m^{-c_{1}} m^{-s} \prod_{k=2}^{\infty}\left(1+\sum_{j=1}^{\infty} \frac{\left(-c_{k} \log m\right)^{j}}{j !} k^{-j s}\right),
\end{aligned}
$$

which holds in the half-plane of absolute convergence of the series $\sum_{k=1}^{\infty} c_{k} k^{-s}$. So $c_{k}=0$ for all $k \geq 2$. Consequently, $\Phi(s)=s+c_{1}$ with $\operatorname{Re} c_{1} \geq 0$.

Conversely, assume that $\Phi(s)=s+c_{1}$ with $\operatorname{Re} c_{1} \geq 0$. Then $C_{\Phi}$ is normal. If $\operatorname{Re} c_{1}>0$, then $C_{\Phi}$ is compact by [5], and hence every invariant subspace of $C_{\Phi}$ contains at least one of eigenvectors. It follows from [17] that $C_{\Phi}$ is reductive. If $\operatorname{Re} c_{1}=0$, then $C_{\Phi}$ is a unitary, and $\left\{n^{-s}\right\}_{n=1}^{\infty}$ are eigenvectors of $C_{\Phi}$. By [17, Theorem 6], there exists a sequence of polynomials $p_{n}(z)$ such that $p_{n}\left(C_{\Phi}\right)$ converges strongly to $C_{\Phi}^{*}$. Hence $C_{\Phi}$ is reductive.

If a non-constant function $f \in \mathcal{H}$ is an eigenvector of a composition operator, then the 2-dimensional subspace span $\{1, f\}$ must be invariant under it. The following actually characterizes 2-dimensional invariant subspaces containing the constants of composition operators.

Theorem 3.5. Let $\Phi(s)=c_{0} s+\sum_{k=1}^{\infty} c_{k} k^{-s}$ be a $c_{0}$-symbol. If there exists a non-constant function $f$ such that $\operatorname{span}\{1, f\}$ is invariant under $C_{\Phi}$, then $c_{0} \leq 1$. Moreover, we have:

(1) If $c_{0}=0$, then $f-f(\alpha)$ is an eigenvector of $C_{\Phi}$ with respect to the eigenvalue $\left(\Phi^{\prime}(\alpha)\right)^{k}$ for some integer $k \geq 1$, where $\alpha$ is the fixed point of $\Phi$ in $\mathbb{C}_{1 / 2}$.

(2) If $c_{0}=1$, then $f-f(+\infty)$ is an eigenvector of $C_{\Phi}$ with respect to the eigenvalue $\ell^{-c_{1}}$ for some integer $\ell \geq 2$.

Proof. Let $c_{0}=0$ and $\alpha$ be the fixed point of $\Phi$. We claim that $g:=f-f(\alpha)$ is an eigenvector of $C_{\Phi}$ with respect to the eigenvalue $\left(\Phi^{\prime}(\alpha)\right)^{k}$ for some integer $k \geq 1$. It is obvious that $g$ is also non-constant and $g \in \operatorname{span}\{1, f\}$. Since span $\{1, f\}$ is invariant under $C_{\Phi}$, we have $C_{\Phi} g=c+d g$ for some $c, d \in \mathbb{C}$. Since $g(\alpha)=0$, then we have $c=0$. Hence $C_{\Phi} g=d g$, i.e., $f-f(\alpha)$ is an eigenvector of $C_{\Phi}$ with respect to the eigenvalue $d$. It is obvious that $d=\left(\Phi^{\prime}(\alpha)\right)^{k}$ for some integer $k \geq 1$ by [3, Theorem 4].

Let $c_{0} \geq 1$ and define the analytic function $\widehat{f}:=f-f(+\infty)$. Then $\widehat{f} \in$ $\operatorname{span}\{1, f\}$ is also non-constant. Since span $\{1, f\}$ is invariant under $C_{\Phi}$, we have 
$C_{\Phi} \widehat{f}(s)=a+b \widehat{f}(s)$ for some constants $a, b \in \mathbb{C}$. Letting Re $s \rightarrow+\infty$ and noting that $\Phi(+\infty)=+\infty$ for $c_{0} \geq 1$, we can get $a=0$ by the fact $\widehat{f}(+\infty)=0$. Hence $C_{\Phi} \widehat{f}(s)=b \widehat{f}(s)$, that is, $\widehat{f}=f-f(+\infty)$ is an eigenvector of $C_{\Phi}$ with respect to the eigenvalue $b$.

We now exclude that $c_{0}>1$. Indeed, if $c_{0}>1$, then $b \in\{0,1\}$ by [3, Theorem 4] and $C_{\Phi} \widehat{f}(s)=b \widehat{f}(s)$ with $\widehat{f}(+\infty)=0$. But this is impossible because $\widehat{f}$ is neither a constant function or zero, which completes the proof.

By the previous theorem, we get that: if $\Phi$ is a $c_{0}$-symbol with $c_{0}>1$, then $C_{\Phi}$ has no 2-dimensional invariant subspaces containing the constants. It is interesting to find other finite dimensional invariant subspaces of $C_{\Phi}$. The following shows the non-existence of non-trivial finite dimensional invariant subspaces.

Theorem 3.6. If $\Phi(s)=c_{0} s+\sum_{k=1}^{\infty} c_{k} k^{-s}$ is a $c_{0}$-symbol with $c_{0}>1$, then $C_{\Phi}$ has no non-trivial finite dimensional invariant subspaces other than the constants.

Proof. Let $\mathcal{M}$ be a non-trivial finite dimensional invariant subspace of $C_{\Phi}$ other than the constants. We first show that $\mathcal{M}$ must contain the constants. According to Theorem 4 in [3], the point spectrum of $C_{\Phi}$ is $\{1\}$ and a computation shows that the eigenvectors corresponding to eigenvalue 1 should be the constants. Since $\mathcal{M}$ is finite dimensional, $\left.C_{\Phi}\right|_{\mathcal{M}}$ has an eigenvector. So 1 is in $\mathcal{M}$. By Lemma 3.1, $\mathbb{C} \in$ Lat $C_{\Phi}^{*}$. So $\mathcal{N}=\mathcal{M} \cap \mathbb{C}^{\perp}$ is a finite dimensional invariant subspace for $C_{\Phi}$, which implies that $\left.C_{\Phi}\right|_{\mathcal{M}}$ has an eigenvalue other than 1 . This yields a contradiction since the point spectrum of $C_{\Phi}$ is $\{1\}$.

\section{REFLEXIVity}

In the sequel, we define $P_{n}$ for $n \geq 1$ on $\mathcal{H}$ as follows: $\left(P_{n} f\right)(s)=a_{n} n^{-s}$ for $f(s)=\sum_{k=1}^{\infty} a_{k} k^{-s} \in \mathcal{H}$. It is clear that $P_{1}$ is the orthogonal projection from $\mathcal{H}$ onto $\mathbb{C}$, so we will also use $P_{\mathbb{C}}$ in place of $P_{1}$. We begin with the following interesting fact.

Theorem 4.1. Let $\Phi$ be a $c_{0}$-symbol with $c_{0}>1$. If a strongly closed unital algebra of operators on $\mathcal{H}$ contains $C_{\Phi}$, then $P_{\mathbb{C}}$ is in the algebra.

Proof. Let $\Phi(s)=c_{0} s+\sum_{k=1}^{\infty} c_{k} k^{-s}$ be a $c_{0}$-symbol with $c_{0}>1$, and let $\mathcal{U}$ be a strongly closed unital algebra of operators on $\mathcal{H}$ containing $C_{\Phi}$.

Fix any $m \geq 1$ and non-zero element $f(s)=\sum_{k=1}^{\infty} a_{k} k^{-s} \in \mathcal{H}$, and set

$$
\mathcal{K}_{m}=\operatorname{span}\left\{1,2^{-s}, \cdots, m^{-s}\right\} .
$$

We claim that $\left(C_{\Phi}^{m}-P_{\mathbb{C}}\right) f \perp \mathcal{K}_{m}$. In fact, it follows from [6] that $\Phi_{m}(s)=$ $c_{0}^{m} s+\sum_{k=1}^{\infty} c_{k}^{(m)} k^{-s}$ with $c_{k}^{(m)} \in \mathbb{C}(k \geq 1)$ for each $m \geq 2$ and $c_{1}^{(m)}=c_{1} \sum_{i=0}^{m-1} c_{0}^{i}$, where the series $\sum_{k=1}^{\infty} c_{k}^{(m)} k^{-s}$ converges uniformly in some (possibly remote) half-plane. Because

$$
\left(C_{\Phi}^{m}-P_{\mathbb{C}}\right) f(s)=\sum_{k=2}^{\infty} a_{k} k^{-\Phi_{m}(s)},
$$

and $n^{c_{0}^{m}} \geq c_{0}^{m}>m$ for $n \geq 2$ and $c_{0}>1$, the expression of $\left(C_{\Phi}^{m}-P_{\mathbb{C}}\right) f(s)$ does not contain any $k^{-s}$ with $k \leq m$. Thus $\left(C_{\Phi}^{m}-P_{\mathbb{C}}\right) f \perp \mathcal{K}_{m}$ for each $m \geq 1$ 
Since $\overline{\cup_{m} \mathcal{K}_{m}}=\mathcal{H}$ and $\left(C_{\Phi}^{m}-P_{\mathbb{C}}\right) f \perp \mathcal{K}_{m},\left(C_{\Phi}^{m}-P_{\mathbb{C}}\right) f$ converges weakly to 0 in $\mathcal{H}$. Thanks to Mazur's theorem (see [14, Theorem 3.13]), there exists a sequence $\left\{h_{N}\right\}$ of convex combinations of $\left(C_{\Phi}^{m}-P_{\mathbb{C}}\right) f$ such that $h_{N} \rightarrow 0$ in $\mathcal{H}$ as $N \rightarrow \infty$, i.e., $h_{N}(s)=\sum_{i=1}^{k_{N}} r_{N_{i}}\left(C_{\Phi}^{N_{i}}-P_{\mathbb{C}}\right) f(s), r_{N_{i}} \geq 0, \sum_{i=1}^{k_{N}} r_{N_{i}}=1$. Then

$$
\sum_{i=1}^{k_{N}} r_{N_{i}} C_{\Phi}^{N_{i}} f(s) \rightarrow P_{\mathbb{C}} f(s), \text { as } N \rightarrow \infty
$$

in the norm of $\mathcal{H}$. By the arbitrariness of $f, P_{\mathbb{C}}$ is in the algebra $\mathcal{U}$.

The following gives a characterization of invariant subspaces of composition operators for $c_{0}$-symbols with $c_{0}>1$.

Corollary 4.2. Let $\Phi$ be a $c_{0}$-symbol with $c_{0}>1$. If $\mathcal{M}$ is an invariant subspace of $C_{\Phi}$, then either $\mathbb{C} \subseteq \mathcal{M}$ or $\mathbb{C} \subseteq \mathcal{M}^{\perp}$.

Proof. Fix any $f \in \mathcal{M}$. By Theorem 4.1, we know that $C_{\Phi}^{n} f$ converges weakly to $P_{\mathbb{C}} f$. Since $\mathcal{M} \in$ Lat $C_{\Phi}$, then $P_{\mathbb{C}} f \in \mathcal{M}$. If $\mathcal{M}$ does not contains the constants, then $P_{\mathbb{C}} f=0$. By the arbitrariness of $f \in \mathcal{M}$, we have $\mathbb{C} \subseteq \mathcal{M}^{\perp}$. So the proof is complete.

Before proceeding to the reflexivity results, we introduce some notation and one useful lemma. If $\Phi(s)=s+i \tau$ with $\tau \in \mathbb{R}$ such that $\left(\frac{n}{m}\right)^{i \tau}$ are not roots of unity for all integers $n \neq m$ and $n, m \geq 1$, then we call $\Phi$ an irrational $c_{0}$-symbol. For example, $\Phi(s)=s+i 2 \pi$ is an irrational $c_{0}$-symbol. If $x$ is a real number, let $\{x\}=x-[x]$, where $[x]$ is the greatest integer less than or equal to $x$. Namely, $\{x\}$ denotes the fractional part of $x$. We recall the following remarkable fact from [11]:

Lemma 4.3. If $\theta$ is an irrational number and $f$ is continuous on $[0,1]$, then

$$
\lim _{n \rightarrow \infty} \frac{1}{n} \sum_{k=0}^{n-1} f(\{k \theta\})=\int_{0}^{1} f(\alpha) d \alpha .
$$

Motivated by Lemma 2.1 and Theorem 2.2 in [10], we give the following two results.

Theorem 4.4. If a strongly closed unital algebra of operators on $\mathcal{H}$ contains a composition operator with an irrational $c_{0}$-symbol, then the algebra contains $P_{n}$ for $n=1,2, \cdots$.

Proof. Fix an $n \geq 1$ and let $\Phi(s)=s+i \tau$ with $\tau \in \mathbb{R}$ such that $\left(\frac{p}{q}\right)^{i \tau}$ are not roots of unity for all integers $p, q \geq 1$ with $p \neq q$. Let $\mathcal{U}$ be a strongly closed unital algebra of operators on $\mathcal{H}$ that contains $C_{\Phi}, C_{n}=n^{i \tau} C_{\Phi}$ and $A_{k}=$ $\frac{1}{k}\left(C_{n}+C_{n}^{2}+\cdots+C_{n}^{k}\right)$. Since $C_{\Phi}$ is a unitary, by the mean ergodic theorem and uniform boundedness principle, $\left\{A_{k}-P_{n}\right\}_{k=1}^{\infty}$ is bounded. It is clear that $E:=\left\{f \in \mathcal{H}: \lim _{k \rightarrow \infty}\left(A_{k}-P_{n}\right) f=0\right\}$ is a closed subspace of $\mathcal{H}$. We now claim 
that $m^{-s} \in E$ for each $m \geq 1$. In fact, we note that

$$
\begin{aligned}
A_{k} m^{-s} & =\frac{1}{k}\left(n^{i \tau} m^{-s-i \tau}+n^{i 2 \tau} m^{-s-i 2 \tau}+\cdots+n^{i k \tau} m^{-s-i k \tau}\right) \\
& =\frac{1}{k}\left[\left(\frac{n}{m}\right)^{i \tau}+\left(\frac{n}{m}\right)^{i 2 \tau}+\cdots+\left(\frac{n}{m}\right)^{i k \tau}\right] m^{-s} .
\end{aligned}
$$

If $m=n$, then $A_{k} n^{-s}=n^{-s}$, and thus $n^{-s} \in E$. If $m \neq n$, then $\theta:=$ $(\tau \log (n / m)) / 2 \pi$ is irrational by our hypothesis. Thus by Lemma 4.3 for $\theta$ and $f(\alpha)=e^{i \alpha 2 \pi}$, we have

$$
\lim _{k \rightarrow \infty} \frac{1}{k}\left[\left(\frac{n}{m}\right)^{i \tau}+\left(\frac{n}{m}\right)^{i 2 \tau}+\cdots+\left(\frac{n}{m}\right)^{i k \tau}\right]=0,
$$

which implies that $m^{-s} \in E$. Consequently, $E$ contains $m^{-s}$ for every $m \geq 1$. Thus $E=\mathcal{H}$, which is equivalent to say $P_{n}$ is the strong limit of $\left\{A_{k}\right\}$. Since $A_{k}$ is in the algebra $\mathcal{U}$ for each $k \geq 1$, it follows that $P_{n}$ is in the algebra for $n=1,2, \cdots$.

Theorem 4.5. If $\Phi$ is an irrational $c_{0}$-symbol, then every strongly closed unital algebra of operators on $\mathcal{H}$ containing $C_{\Phi}$ is reflexive.

Proof. Let $\mathcal{U}$ be any strongly closed unital algebra of operators on $\mathcal{H}$ containing $C_{\Phi}$. Then $\mathcal{U}$ contains $P_{n}$ for $n=1,2, \cdots$ by Theorem 4.4. Suppose $B \in B(\mathcal{H})$ such that Lat $\mathcal{U} \subseteq$ Lat $B$. For arbitrary $n, B n^{-s}$ is in the cyclic subspace of $\mathcal{U}$ determined by $n^{-s}$, and thus there is a sequence $\left\{A_{k}\right\}$ in $\mathcal{U}$ such that $B n^{-s}=$ $\lim _{k \rightarrow \infty} A_{k} n^{-s}$. It follows that $B P_{n}$ is the strong limit of $\left\{A_{k} P_{n}\right\}_{k=1}^{\infty}$, and hence $B P_{n} \in \mathcal{U}$.

Let $\sigma_{k}$ be the $k$-th Cesàro mean of the series $\sum_{m=1}^{\infty} P_{m}$, i.e., $\sigma_{k}=\sum_{m=1}^{k} \frac{k+1-m}{k+1} P_{m}$. Let $\widetilde{P}_{N}$ be the $N$-th partial sum of $\sum_{m=1}^{\infty} P_{m}$, i.e., $\widetilde{P}_{N}=\sum_{m=1}^{N} P_{m}$. It is clear that all $\sigma_{k}(k \geq 1)$ and $\widetilde{P}_{N}(N \geq 1)$ are in $\mathcal{U}$. Note that $\lim _{k \rightarrow \infty} \sigma_{k} P=P$ for any polynomial $P$ with respect to $\left\{n^{-s}\right\}_{n=1}^{\infty}$. Also for any fixed $f(s)=\sum_{k=1}^{\infty} a_{k} k^{-s} \in \mathcal{H}$, and $\epsilon>0$, it is trivial that there is an integer $N_{0}$ such that for each $N \geq$ $N_{0}$, we have $\left\|\widetilde{P}_{N} f-f\right\|<\epsilon / 2$. Further we can find an integer $k_{N}$ such that $\left\|\sigma_{k_{N}} \widetilde{P}_{N} f-\widetilde{P}_{N} f\right\|<\epsilon / 2$. Therefore $\sigma_{k_{N}} \widetilde{P}_{N} f$ converges to $f$ in $\mathcal{H}$ as $N \rightarrow \infty$. It follows that $B \sigma_{k_{N}} \widetilde{P}_{N}$ converges strongly to $B$ in $\mathcal{H}$. Since $B \sigma_{k_{N}} \in \mathcal{U}$ and $\widetilde{P}_{N} \in \mathcal{U}$, then $B \sigma_{k_{N}} \widetilde{P}_{N} \in \mathcal{U}$. And thus $B \in \mathcal{U}$, which completes the proof.

Next we give a strongly closed unital algebra of operators on $\mathcal{H}$ generated by certain sets of composition operators such that the non-trivial common invariant subspace of the algebra is $\mathbb{C}$. For convenience, we will denote by Prime the set of all prime numbers.

Theorem 4.6. Let $\Phi$ be an irrational $c_{0}$-symbol, and $\Gamma(s)=c_{1}+\sum_{p \in \text { Prime }} c_{p} p^{-s}$ a $c_{0}$-symbol with $c_{p} \neq 0$. Then the strongly closed unital algebra of operators on $\mathcal{H}$ generated by $C_{\Phi}$ and $C_{\Gamma}$ is $\operatorname{Alg}\{\{0\}, \mathbb{C}, \mathcal{H}\}$.

Proof. Let $\mathcal{U}$ denote the strongly closed unital algebra of operators on $\mathcal{H}$ generated by $C_{\Phi}$ and $C_{\Gamma}$, and let $\mathcal{M}$ be an arbitrary invariant subspace of $\mathcal{U}$. If $\mathcal{M} \neq\{0\}$, then we claim that $\mathcal{M}$ must contain the constants $\mathbb{C}$. In fact, since $\mathcal{M} \neq\{0\}$, then there is a non-zero function $f(s)=\sum_{k=1}^{\infty} a_{k} k^{-s} \in \mathcal{M}$. If $a_{1} \neq 0$, 
then $a_{1}=P_{1} f(s) \in \mathcal{M}$, i.e., $\mathbb{C} \subseteq \mathcal{M}$. If $a_{1}=0$, then $f(s)=\sum_{k=\ell}^{\infty} a_{k} k^{-s}$ for $a_{\ell} \neq 0$ and $\ell \geq 2$. Since $\mathcal{M}$ is invariant under $P_{\ell}$, then $\ell^{-s} \in \mathcal{M}$. Due to

$$
\begin{aligned}
C_{\Gamma} \ell^{-s} & =\ell^{-\Gamma(s)}=\ell^{-c_{1}} \ell^{-\sum_{p \in \text { Prime }} c_{p} p^{-s}} \\
& =\ell^{-c_{1}} \prod_{p \in \text { Prime }}\left(1+\sum_{j=1}^{\infty} \frac{\left(-c_{p} \log \ell\right)^{j}}{j !} p^{-j s}\right),
\end{aligned}
$$

we have $P_{1} C_{\Gamma} \ell^{-s}=\ell^{-c_{1}} \neq 0$. Again $C_{\Gamma} \mathcal{M} \subseteq \mathcal{M}$ implies that $P_{1} C_{\Gamma} \mathcal{M} \subseteq P_{1} \mathcal{M} \subseteq$ $\mathcal{M}$. Therefore $\ell^{-c_{1}} \in \mathcal{M}$, which implies the claim.

Moreover, if $\mathcal{M} \neq \mathbb{C}$, then there exists some $n_{0} \geq 2$ such that $n_{0}^{-s} \in \mathcal{M}$. Since $P_{n} \in \mathcal{U}$ for every $n \geq 1$ by Theorem 4.4 and $C_{\Gamma} \mathcal{M} \subseteq \mathcal{M}$, then $P_{n} C_{\Gamma} n_{0}^{-s} \in \mathcal{M}$ for all $n \geq 1$. We know that each positive integer $n$ can be uniquely expressed as

$$
n=p_{1}^{\alpha_{1}} p_{2}^{\alpha_{2}} \cdots p_{\ell}^{\alpha_{\ell}},
$$

where $p_{1}<p_{2}<\cdots<p_{\ell} \in$ Prime and $\alpha_{1}, \alpha_{2}, \cdots, \alpha_{\ell}$ are positive integers. Because

$$
C_{\Gamma} n_{0}^{-s}=n_{0}^{-c_{1}} \prod_{p \in \text { Prime }}\left(1+\sum_{j=1}^{\infty} \frac{\left(-c_{p} \log n_{0}\right)^{j}}{j !} p^{-j s}\right),
$$

we can get the coefficient of $n^{-s}$ in (4.1) as follows

$$
a_{n}:=n_{0}^{-c_{1}} \frac{\left(-c_{p_{1}} \log n_{0}\right)^{\alpha_{1}}}{\alpha_{1} !} \frac{\left(-c_{p_{2}} \log n_{0}\right)^{\alpha_{2}}}{\alpha_{2} !} \cdots \frac{\left(-c_{p_{\ell}} \log n_{0}\right)^{\alpha_{\ell}}}{\alpha_{\ell} !} .
$$

It is clear that $a_{n} \neq 0$. Note that $P_{n} C_{\Gamma} n_{0}^{-s}=a_{n} n^{-s}$, which gives that $n^{-s} \in$ $\mathcal{M}$. Thus $\operatorname{span}\left\{n^{-s}: n \geq 1\right\} \subseteq \mathcal{M}$ and $\mathcal{M}=\mathcal{H}$. We now get that Lat $\mathcal{U}=$ $\{\{0\}, \mathbb{C}, \mathcal{H}\}$. By Theorem 4.5, we know that the algebra $\mathcal{U}$ is reflexive. This immediately gives that $\mathcal{U}=\operatorname{Alg} \operatorname{Lat} \mathcal{U}=\operatorname{Alg}\{\{0\}, \mathbb{C}, \mathcal{H}\}$, which completes the proof.

Interestingly, the following shows that the subalgebra of $B(\mathcal{H})$ generated by certain sets of composition operators and their adjoints is $B(\mathcal{H})$.

Corollary 4.7. Let $\Phi$ be an irrational $c_{0}$-symbol, and let $\Gamma(s)=\sum_{k=1}^{\infty} c_{k} k^{-s}$ be a $c_{0}$-symbol. Then the strongly closed unital algebra of operators on $\mathcal{H}$ generated by $C_{\Phi}, C_{\Gamma}$ and their adjoints equals $B(\mathcal{H})$.

Proof. Let $\mathcal{U}$ be the strongly closed unital algebra of operators on $\mathcal{H}$ generated by $C_{\Phi}, C_{\Gamma}$ and their adjoints, and let $M \neq\{0\}$ be any invariant subspace of $\mathcal{U}$. By the proof of Theorem 4.6, we know that $\mathbb{C} \subseteq M$. Since $M$ is invariant under $C_{\Gamma}^{*}, C_{\Gamma}^{*} 1 \in M$. Again $C_{\Gamma}^{*} 1=\sum_{n=1}^{\infty} n^{-\overline{c_{1}}} n^{-s}$ by Lemma 3.1 and $P_{n} C_{\Gamma}^{*} 1 \in M$ for all $n \geq 1$ by Theorem 4.4, we have $n^{-s} \in M$ for all $n \geq 1$, i.e., $\mathcal{H}=M$. Therefore Lat $\mathcal{U}=\{\{0\}, \mathcal{H}\}$. Corollary 8.5 in [13] now gives the desired assertion $\mathcal{U}=B(\mathcal{H})$.

Algebraic composition operators on Hardy and Bergman spaces were studied by many authors. Especially, Mahvidi showed that the non-existence of non-trivial algebraic composition operators on $H^{2}(\mathbb{D})$ by using their invariant subspaces in [8]. At the end of this section we extend Mahvidi's result to the Dirichlet series case. 
Theorem 4.8. Let $\Phi(s)=c_{0} s+\sum_{k=1}^{\infty} c_{k} k^{-s}$ be a $c_{0}$-symbol. Then on $\mathcal{H}$ the following statements hold.

(1) If $c_{0}=0$ and $\left\{\Phi^{\prime}(\alpha)^{n}\right\}_{n=0}^{\infty}$ is infinite for the fixed point $\alpha$ of $\Phi$ in $\mathbb{C}_{1 / 2}$, then $C_{\Phi}$ is not algebraic.

(2) If $c_{0}=1$ and $c_{1} \neq 0$, then $C_{\Phi}$ is not algebraic.

(3) If $c_{0}>1$, then $C_{\Phi}$ is not algebraic.

Proof. (1) If $C_{\Phi}$ is algebraic on $\mathcal{H}$, then so is $C_{\Phi}^{*}$. Thus there exists a polynomial $P(z)=z^{n}+p_{n-1} z^{n-1}+\cdots+p_{1} z+p_{0}$ such that $P\left(C_{\Phi}^{*}\right)=0$ on $\mathcal{H}$. So

$$
\left(C_{\Phi}^{*}\right)^{n} K_{\alpha}^{[m]}+p_{n-1}\left(C_{\Phi}^{*}\right)^{n-1} K_{\alpha}^{[m]}+\cdots+p_{1} C_{\Phi}^{*} K_{\alpha}^{[m]}+p_{0} K_{\alpha}^{[m]}=0
$$

for each $m \geq 0$, where $K_{\alpha}^{[m]}$ denotes the $m$-th derivative of $K_{\alpha}$. Since the coefficient of $K_{\alpha}^{[m]}$ in the expansion of the left hand side of the equation (4.2) is

$$
\left(\left[\Phi^{\prime}(\alpha)\right]^{m}\right)^{n}+p_{n-1}\left(\left[\Phi^{\prime}(\alpha)\right]^{m}\right)^{n-1}+\cdots+p_{1}\left[\Phi^{\prime}(\alpha)\right]^{m}+p_{0}
$$

for each $m \geq 0$. So $\left[\Phi^{\prime}(\alpha)\right]^{m}$ for all $m \geq 0$ are the roots of the equation $P(z)=0$. But the polynomial $P(z)$ has at most $n$ zeros, which leads to a contradiction. Therefore $C_{\Phi}$ is not algebraic.

(2) If $c_{0}=1$, then $\Phi_{n}(s)=s+n c_{1}+\sum_{k=2}^{\infty} c_{k}^{(n)} k^{-s}$ with some $c_{k}^{(n)} \in \mathbb{C}(k \geq 2)$ for each $n \geq 2$, where the series $\sum_{k=2}^{\infty} c_{k}^{(n)} k^{-s}$ converges uniformly in some (possibly remote) half-plane. Suppose that there is a polynomial $P(z)=z^{n}+p_{n-1} z^{n-1}+$ $\cdots+p_{1} z+p_{0}$ such that $P\left(C_{\Phi}\right)=0$ on $\mathcal{H}$, then

$$
m^{-\Phi_{n}(s)}+p_{n-1} m^{-\Phi_{n-1}(s)}+\cdots+p_{1} m^{-\Phi(s)}+p_{0} m^{-s}=0 .
$$

Note that

$$
m^{-\Phi_{j}(s)}=m^{-s} m^{-j c_{1}} \prod_{k=2}^{\infty}\left(1+\sum_{i=1}^{\infty} \frac{\left(-c_{k}^{(j)} \log m\right)^{i}}{i !} k^{-i s}\right)
$$

in some (possibly remote) half-plane for $m \geq 1$ and $j \geq 1$. Considering the coefficient of $m^{-s}$ in the left hand side of (4.3), we get

$$
m^{-n c_{1}}+p_{n-1} m^{-(n-1) c_{1}}+\cdots+p_{1} m^{-c_{1}}+p_{0}=0,
$$

that is, $m^{-c_{1}}$ is a root of $P(z)=0$. Then by the arbitrariness of $m$, the polynomial $P$ has zeros $1,2^{-c_{1}}, 3^{-c_{1}}, \cdots, m^{-c_{1}}, \cdots$. Note that, if $\operatorname{Re} c_{1} \neq 0$, then $m^{-c_{1}}$ are mutually distinct for all $m \geq 1$; if $\operatorname{Re} c_{1}=0$ but $\operatorname{Im} c_{1} \neq 0$, then $m^{-c_{1}}=$ $m^{-i \operatorname{Im} c_{1}}=e^{-i \operatorname{Im} c_{1} \log m}$ are dense in the unit circle, by Lemma 3.5 in [7]. In short, the polynomial $P$ has infinitely many zeros whenever $c_{1} \neq 0$. This contradiction implies that $C_{\Phi}$ is not algebraic.

(3) If $c_{0}>1$, by the proof of Theorem $4.1,\left(C_{\Phi}^{*}\right)^{n} f$ converges weakly to $P_{\mathbb{C}} f$ in $\mathcal{H}$. Thus

$$
\left\langle C_{\Phi_{n}}^{*} K_{w}, g\right\rangle \rightarrow\left\langle P_{\mathbb{C}} K_{w}, g\right\rangle
$$

for each fixed $w \in \mathbb{C}_{1 / 2}$ and $g \in \mathcal{H}$, as $n \rightarrow \infty$. Note that $P_{\mathbb{C}} K_{w}(s)=1$. Thus

$$
m^{-\overline{\Phi_{n}(w)}}=\left\langle K_{\Phi_{n}(w)}, m^{-s}\right\rangle \rightarrow\left\langle 1, m^{-s}\right\rangle= \begin{cases}1, & m=1 \\ 0, & m>1\end{cases}
$$


as $n \rightarrow \infty$. Therefore the sequence $\left\{\operatorname{Re} \Phi_{n}(w)\right\}_{n=0}^{\infty}$ tends to $+\infty$. If $C_{\Phi}$ on $\mathcal{H}$ is algebraic, then so is $C_{\Phi}^{*}$. Hence, all cyclic subspaces of $C_{\Phi}^{*}$ are finite dimension. But the cyclic subspace $\operatorname{span}\left\{\left(C_{\Phi}^{*}\right)^{n} K_{w}: n \geq 0\right\}=\operatorname{span}\left\{K_{\Phi_{n}(w)}: n \geq 0\right\}$ of $C_{\Phi}^{*}$ is infinite dimension, because $\left\{K_{w}\right\}$ are linearly independent for $w \in \mathbb{C}_{1 / 2}$ with distinct real parts. This contradiction completes the proof.

Acknowledgment. This research is partially supported by National Science Foundation of China $(11271293,11431101,11471251)$ and the Research Fund for the Doctoral Program of Higher Education of China (2014201020205).

\section{REFERENCES}

1. M. Bailleul, Composition operators on weighted Bergman spaces of Dirichlet series, J. Math. Anal. Appl.426 (2015), 340-363.

2. F. Bayart, Hardy spaces of Dirichlet series and their composition operators, Monatsh. Math.136 (2002), 203-236.

3. F. Bayart, Compact composition operators on a Hilbert space of Dirichlet series, Illinois J. Math.47 (2003), 725-743.

4. C. Cowen and B. MacCluer, Composition operators on spaces of analytic functions, CRC Press, Boca Raton, 1995.

5. C. Finet, H. Queffélec and A. Volberg, Compactness of composition operators on a Hilbert space of Dirichlet series, J. Funct. Anal.211 (2004), 271-287.

6. J. Gordon and H. Hedenmalm, The composition operators on the space of Dirichlet series with square summable coefficients, Michigan Math. J.46 (1999), 313-329.

7. B. Hu, L. Khoi and R. Zhao, Topological structure of the space of composition operators on Hilbert spaces of Dirichlet series, preprint.

8. A. Mahvidi, Invariant subspaces of composition operators, J. Operator Theory 46 (2001), 453-476.

9. V. Matache, On the minimal invariant subspaces of the hyperbolic composition operator, Proc. Amer. Math. Soc. 119 (1993), 837-841.

10. E. Nordgren, P. Rosenthal and F. Wintrobe, Invertible composition operators on $H^{p}$, J. Funct. Anal. 73 (1978), 324-344.

11. K. Peterson, Ergodic Theory, Cambridge University Press, Cambridge, 1983.

12. H. Queffélec and K. Seip, Approximation numbers of composition operators on the $H^{2}$ space of Dirichlet series, J. Funct. Anal. 268 (2015), 1612-1648.

13. H. Radjavi and P. Rosenthal, Invariant subspaces, Springer-Verlag, New York-HeidelbergBerlin, 1973.

14. W. Rudin, Functional analysis, McGraw-Hill, New York, 1973.

15. J. Shapiro, Composition operators and classical function theory, Springer, New York, 1993.

16. M. Wang and X. Yao, Some properties of composition operators on Hilbert spaces of Dirichlet series, Complex Var. Elliptic Equ. 60 (2015), 992-1004.

17. J. Wermer, On invariant subspaces of normal operators, Proc. Amer. Math. Soc. 33 (1972) 415-419.

18. K. Zhu, Operator theory in function spaces, Second edition. Mathematical Surveys and Monographs, 138. American Mathematical Society, Providence, RI, 2007.

School of Mathematics and Statistics, Wuhan University, Wuhan 430072, China.

E-mail address: mfwang.math@whu.edu.cn

E-mail address: xxyao.math@whu.edu.cn 